\title{
Agriculture urbaine et autogestion à Cuba
} Urban farming and self-management in Cuba Agricultura urbana y autogestión en Cuba

\section{Ingrid Hanon}

Numéro 337, juillet 2015

L'ESS en Amérique latine : de nouvelles pratiques

New Practices in the Social and Solidarity Economy in Latin America

URI : https://id.erudit.org/iderudit/1032525ar

DOI : https://doi.org/10.7202/1032525ar

Aller au sommaire du numéro

\section{Éditeur(s)}

Association Recma

\section{ISSN}

1626-1682 (imprimé)

2261-2599 (numérique)

Découvrir la revue

\section{Citer cet article}

Hanon, I. (2015). Agriculture urbaine et autogestion à Cuba. Revue internationale de l'économie sociale, (337), 84-99.

https://doi.org/10.7202/1032525ar
Résumé de l'article

Cet article a pour objet l'étude de l'agriculture cubaine depuis la révolution de 1959, et notamment son émergence au cours des années 90. L’agriculture urbaine a contribué autant à assurer la sécurité alimentaire du pays qu’à construire une économie moins centralisée et plus autonome par rapport au pouvoir politique à Cuba, grâce au développement d'unités économiques démocratiques et autogérées, fondées sur les principes de solidarité, d'attention au bien-être de la communauté et de respect de la nature. Cette nouvelle forme d'économie sociale se différencie des entreprises capitalistes, dont l'objectif est la concentration de pouvoirs et l'accumulation de richesses et de capital à travers l'exploitation du travail et de la nature. Elle se distingue également des traditionnelles entreprises étatiques cubaines, au sein desquelles les initiatives individuelles et la créativité des travailleurs sont limitées. 


\title{
AGRICULTURE URBAINE ET AUTOGESTION À CUBA
}

\author{
par Ingrid Hanon*
}

\footnotetext{
* Faculté d'économie de l’université de Bordeaux (France), universitédeQuilmes (Argentine).
}

Cet article a pour objet l'étude de l'agriculture cubaine depuis la révolution de 1959, et notamment son émergence au cours des années 90 . L'agriculture urbaine a contribué autant à assurer la sécuritéalimentaire du pays qu'à construire une économie moins centralisée et plus autonome par rapport au pouvoir politique à Cuba, grâce au développement d'unités économiques démocratiques et autogérées, fondées sur les principes de solidarité, d'attention au bien-être de la communauté et de respect de la nature. Cette nouvelle forme d'économie sociale se différencie des entreprises capitalistes, dont l'objectif est la concentration de pouvoirs et l'accumulation de richesses et de capital à travers l'exploitation du travail et de la nature. Elle se distingue également des traditionnelles entreprises étatiques cubaines, au sein desquelles les initiatives individuelles et la créativité des travailleurs sont limitées.

\section{Urban farming and self-management in Cuba}

This article looks at Cuban agriculture since the 1959 Revolution and, in particular, its rise in the 1990s. Urban farming has helped guarantee the country's food security and create a less centralised and more independent economy in relation to the Cuban authorities thanks to the development of democratic, self-managed, economic units based on the principles of solidarity, concern for the well-being of the community, and the respect of nature. This new form of social economy differs from capitalist enterprises whose objective is the concentration of power and the accumulation of wealth and capital through the exploitation of labour and nature. It also differs from traditional state-owned enterprises in Cuba where there is little scope for individual initiative and creativity by workers.

\section{Agricultura urbana y autogestión en Cuba}

Este artículo tiene como finalidad el estudio de la agricultura cubana desde la Revolución de 1959 y, en particular, el surgimiento de la agricultura urbana en los años 90. La agricultura urbana ha contribuido tanto a la seguridad alimentaria del país como a la construcción de una economía menos centralizada y más autónoma del poder político en Cuba, gracias al desarrollo de unidades económicas democráticas y auto-gestionadas creadas sobre los principios de la solidaridad, la preocupación por el bienestar de la comunidad y el respeto de la naturaleza. Esta nueva forma de economía social se diferencia de la empresa capitalista cuyo objetivo es la concentración de poder y la acumulación de riquezas. Sin embargo, ella se diferencia también de las tradicionales empresas estatales cubanas dentro de las cuales las iniciativas individuales y la creatividad de los trabajadores son limitadas. 
Bien qu'ayant un système économique socialiste, Cuba met en place, des années 60 aux années 90, une politique de modernisation de l'agriculture comparable à celle des pays du monde capitaliste: une agriculture industrielle caractérisée par la forte consommation d'intrants externes (la motorisation, la mécanisation, les engrais chimiques, etc.), la spécialisation agricole dans un nombre réduit de produits d'exportation au détriment de la production alimentaire vivrière et la production de monocultures à grande échelle aux mains des fermes d'Etat.

Cependant, au cours des années 90, la chute de l'Union soviétique remet en question le modèle agricole conventionnel. Du fait de la forte dépendance externe, le secteur agricole de l'île est l'un des plus gravement touchés, ce qui menace la sécurité alimentaire du pays.

Il faut donc trouver des réponses rapides aux besoins alimentaires de la population. C'est ainsi que de multiples alternatives vont naître à Cuba, afin de réactiver la production agricole et de pallier les effets de la crise de la " période spéciale », parmi lesquelles l'agriculture urbaine, conçue sous l’angle écologique.

L'article propose, dans une première partie, une brève description des principales caractéristiques du système agroalimentaire cubain depuis la révolution jusqu'aux années 90 pour mieux comprendre l'importance et le rôle de l'agriculture urbaine dans le pays.

Nous aborderons ensuite l'essor de cette dernière, sa consolidation dans l'île et les différentes formes d'organisation qu'elle adopte.

Enfin, nous identifierons les apports de l'agriculture urbaine - ses multiples formes productives non étatiques et ses modes de gestion - à l'actualisation du socialisme cubain à partir des principes de l'économie sociale et solidaire, dont elle fait partie.

\section{L'organisation de l'agriculture cubaine après la révolution}

\section{Modernisation de l'agriculture et dépendance externe}

Dès son installation en 1959, le gouvernement révolutionnaire s'engage dans une transformation radicale du paysage agricole de l'île, avec l'objectif d'en finir avec la pauvreté rurale, d'améliorer les conditions de vie de la petite paysannerie et de limiter la concentration de la terre. Deux réformes agraires, mises en place afin de distribuer la terre à ceux qui la travaillent, bénéficient à plus de 100000 paysans (Gutelman, 1963). L'Etat garantit l'assistance technique et des prêts bancaires à faible intérêt pour les petits agriculteurs. Les travailleurs agricoles obtiennent des salaires plus élevés et une meilleure stabilité d'emploi. D’importants investissements dans les écoles, les centres de santé, l'électrification, les routes ou encore les logements sont réalisés dans les zones rurales et une campagne massive d’alphabétisation est mise en œuvre en 1961 (Karnoouh, 2007), ce qui favorise la mobilité sociale des enfants des familles paysannes vers d'autres secteurs économiques, propres aux zones urbaines (Marzin, 2013). Le gouvernement ne distribue toutefois pas toutes les terres, par crainte d'une diminution de la productivité après la répartition de la terre entre de nombreux minifundia (Gutelman, 1967). Une partie des anciens latifundia reste donc dans les mains de l'Etat, ce qui donne lieu à la consolidation 
d'un important secteur étatique de l'agriculture cubaine. A la fin des années 80 , celui-ci contrôle plus de $70 \%$ des terres agricoles du pays (Guevara, 2009).

De plus, après un éphémère programme de diversification agricole, l'île revient vers la production de canne à sucre comme moteur du développement du pays, à la suite de l'intensification des échanges commerciaux avec l'URSS, qui s'engage à acheter le sucre cubain à un prix plus favorable que celui du marché international (Gutelman, 1965; Nova, 2001).

Le retour à la culture de la canne à sucre et la production à grande échelle dans les fermes d'Etat favorisent la modernisation de l'agriculture. Ainsi, l'agriculture conventionnelle se consolide à Cuba. Elle se caractérise par la spécialisation agricole, l'existence de grands espaces de monocultures, l'emploi massif de produits chimiques, la mécanisation agricole, l'irrigation à grande échelle et la forte consommation d'énergie (pétrole) dans le but d'augmenter la productivité (Gonzalez, 2003).

Le bilan des trente premières années de la révolution, très positif en termes de progrès sociaux, l'est moins en termes de dépendance externe, de vulnérabilité alimentaire et de dégradation de l'environnement. La production agricole du pays est devenue fortement dépendante des importations d'intrants et de machines ( $48 \%$ des engrais chimiques, $82 \%$ de pesticides et $97 \%$ de la nourriture des animaux) à la fin des années 80 (Gonzalez, 2003). Néanmoins, malgré l'introduction massive des machines et de l'agro-chimie et l'exploitation d'immenses surfaces pour profiter des effets d'économie d'échelle et augmenter les rendements agricoles, les résultats obtenus sont loin d'être proportionnels aux capitaux investis (Raymond, 2002), principalement dans les fermes d'Etat surdimensionnées. Au contraire, avec une utilisation moindre d'intrants agricoles, les petits agriculteurs du secteur privé sont plus productifs que le secteur agricole de l'Etat (Medea et al., 1987). En outre, même si la faim a été éradiquée, la vulnérabilité alimentaire reste forte. L'étroite relation économique de l'île avec les pays socialistes d'Europe de l'Est conduit à la spécialisation agricole dans un nombre réduit de matières premières, au détriment de la production vivrière.

Nonobstant les effets négatifs sur la sécurité alimentaire du pays, la dégradation de l'environnement et le renforcement de la dépendance externe du modèle agricole conventionnel issue de la révolution verte, Cuba ne prend véritablement conscience des enjeux de ce système agricole et alimentaire qu'après la chute de l'URSS.

\section{La période spéciale et son impact sur l'agriculture et l'alimentation dans l'île}

Les années 90 mettent en évidence la fragilité du modèle agricole conventionnel à Cuba. La disparition du principal partenaire économique del'île, qui représentait $70 \%$ deséchanges commerciaux du pays en 1989, conduit à une crise sans précédent dans l'histoire de la révolution, connue sous le nom de "el periodo especial en tiempos de $p a z^{(1)}$ »; le secteur agricole est l'un des plus gravement touchés (Herrera, 2005). Les importations d'engrais chimiques chutent de $77 \%$, des pesticides de $60 \%$ et de pétrole, indispensable pour faire fonctionner

\footnotetext{
(1) «La période spéciale en temps de paix ", car l'île se retrouve dans une situation de pénurie et d'austérité similaire à celle d'un contexte d'économie de guerre.
} 
les machines, de moitié. Les importations d'aliments pour l'élevage diminuent également de 70 \% (Rosset et Medea, 1994; Altieri et al., 1999).

La chute de ces importations conduit à une réduction dramatique de la production agricole du pays, principalement dans le secteur sucrier, mais aussi dans les secteurs du tabac et des agrumes, indispensables pour l'obtention de devises. De même, la production agricole nationale destinée à la consommation locale, déjà très faible avant la crise, car ne pouvant satisfaire que $40 \%$ des besoins alimentaires, baisse encore plus (Habel, 2003). Non seulement la production agricole domestique se réduit, mais aussi l'importation des aliments en provenance de l'ex-Union soviétique, qui assurait $66 \%$ des besoins alimentaires de la population cubaine (Argaillot, 2014). De plus, le manque de pétrole affecte également les autres services essentiels pour garantir l'alimentation tels que le transport et la réfrigération.

Ainsi, ce pays, dans lequel le problème de la faim a été éliminé dès le début de la révolution et où l'accès égalitaire et universel à l'alimentation est

(2) En 1962, le gouvernement cubain a mis en place un système de rationnement capable de garantir à l'ensemble de la population l'accès égalitaire, universel et à de faibles coûts aux produits alimentaires de base.

3) Système d'approvisionnement d'aliments introduit à Cuba en 1963 pour garantir à tous l'accès à des produits de base à très bas prix sur une période d'un mois.

(4) Réouverture des marchés paysans, création d'unidades básicas de producción cooperativa (UBPC), développement de l'agriculture organique ou semi-organique, revalorisation des connaissances paysannes, réutilisation de la traction animale, etc.

garanti depuis $1962^{(2)}$, se retrouve pour la première fois depuis des décennies avec des taux de consommation en calories et en protéines en dessous des standards de consommation minimale établis par l'Organisation des Nations unies pour l'alimentation et l'agriculture (FAO, 2014; Nova, 2001; Roux, 1999). Selon cette institution internationale, les Cubains sont en effet passés d'une consommation moyenne de 3004 calories par jour en 1989 à 2323 en 1993, l'année la plus dure de la période spéciale. Cette situation est en outre aggravée par le renforcement de l'embargo des EtatsUnis (loi Torricelli en 1992; loi Helms-Burton en 1996), qui rend encore plus difficile l'accès aux denrées alimentaires.

Selon Janette Habel (2003), lors de la période spéciale, la livreta ${ }^{(3)}$ ne couvre plus que dix à quinze jours d'approvisionnement alimentaire, et la population doit acheter dans les agromercados le reste des produits dont elle a besoin, souvent à des prix très élevés.

Face à la crise qui menace le pays, le modèle de l'agriculture conventionnelle est remis en question. La nation cubaine se lance alors dans la recherche d'alternatives ${ }^{(4)}$ capables d'assurer l'alimentation de la population et d'accroître la production agricole pour la consommation interne et pour l'exportation, tout en limitant l'utilisation d'intrants externes (Douzant-Rosenfeld, 1999). La plupart de ces politiques s'orienteront vers la décentralisation et la désétatisation de l'agriculture (Marzin, 2013).

C'est de cette manière que le modèle agricole de monoculture à grande échelle est remplacé par un modèle agricole de taille plus réduite, plus diversifié, moins consommateur d'énergie et plus respectueux de l'environnement.

\section{L’agriculture urbaine à Cuba}

\section{Emergence de l'agriculture urbaine}

Lors de la crise de la période spéciale, la pénurie alimentaire à Cuba affecte principalement les zones urbaines, fortement dépendantes des zones rurales pour assurer leur propre alimentation. Ravitailler les villes depuis les zones 
rurales devient difficile du fait des coupures d'approvisionnement de pétrole pour réfrigérer, transporter, stocker et distribuer la nourriture: seulement $50 \%$ à $60 \%$ de la production agricole du pays parvient aux ménages cubains (Gonzalez Novo, 2000). Une grande partie des aliments est gaspillée dans la longue chaîne entre la production et la vente aux consommateurs urbains (du fait d'un système défectueux de transport et de stockage des aliments), et la plupart des fruits et légumes qui arrivent sur la table des Cubains sont en mauvais état.

Il faut donc trouver des réponses rapides aux besoins alimentaires. C'est pour cela, afin de garantir la sécurité alimentaire de la population des villes, qui représente $73 \%$ de la population cubaine en 1993 (chiffres de l'Office national de statistiques de Cuba, $\left.\mathrm{ONE}^{(5)}\right)$, qu'émerge l'agriculture urbaine. Ce mouvement, fruit d'une initiative populaire, naît de manière spontanée parmi la population des villes, principale-

(5) www.one.cu. ment à La Havane, pour augmenter l'offre domestique de nourriture aux citadins en profitant des espaces vacants dans les villes (Altieri et al., 1999). Par ailleurs, pour des provinces comme La Havane, Matanzas, Cienfuegos ou Camagüey avec un pourcentage de population urbaine de plus de $70 \%$, la relocalisation de la production agricole à petite échelle dans les centres urbains permet non seulement de répondre aux besoins alimentaires, mais aussi de profiter de la grande disponibilité de main-d'œuvre dans ces régions, ce qui entraîne l'émergence de " néo-agriculteurs » (Marzin, 2013), pas nécessairement en lien avec la paysannerie traditionnelle.

Bien que les citadins ne se soient jamais préoccupés de la production agricole, car l'Etat révolutionnaire garantit depuis toujours l'accès à la nourriture par des carnets de rationnement (Murphy, 1999), la rupture soudaine des aliments fournis par le gouvernement et les prix exorbitants du marché noir poussent les habitants des villes à cultiver eux-mêmes des plantes alimentaires dans leurs cours, sur leurs balcons, leurs toits ou dans leurs jardins. Les familles qui ont la chance d'avoir leur propre jardin le reconvertissent en parcelle productive. D’autres récupèrent des terrains vagues pour installer des potagers (Douzant-Rosenfeld, 1999). Ce mouvement autonome et populaire est ainsi à l'origine de l'émergence de l'agriculture urbaine à Cuba.

\section{L'appui de l'Etat à l'agriculture urbaine}

Née de manière spontanée, l'agriculture urbaine devient rapidement une stratégie gouvernementale afin d'augmenter l'offre domestique de nourriture pour la consommation locale et la vente sur les marchés de proximité. Ce sont les municipalités qui, les premières, appuient le mouvement en distribuant des parcelles de jardin de moins de 0,5 hectare aux personnes qui le souhaitent, dans l'objectif de favoriser les cultures vivrières de proximité. Le mouvement procure donc rapidement des légumes frais pour les populations des zones urbaines et périurbaines. C'est alors que le gouvernement, confronté à l'impossibilité d'importer des produits alimentaires, envisage l'agriculture urbaine comme une stratégie au niveau national pour garantir la sécurité alimentaire de la population et décide de rejoindre ce mouvement. 
Dès 1994, le mouvement de l'agriculture urbaine devient une priorité nationale et le département de l'agriculture urbaine au sein du ministère de l'Agriculture (Minag) est créé. Le Minag élabore alors un programme d'agriculture urbaine (Murphy, 1999), ayant pour but de garantir l'accès à la terre aux personnes qui le souhaitent pour la production vivrière, de créer des systèmes de soutien et de conseil aux jardiniers, d'organiser de nouveaux points de vente pour les agriculteurs, de promouvoir la recherche et le développement de l'agriculture urbaine autour des techniques organiques, etc. D'une réponse spontanée à un besoin essentiel, l'agriculture urbaine devient une politique nationale.

Ce programme est mis en place en premier lieu à La Havane, car il faut approvisionner cette métropole de plus de 2,5 millions de consommateurs. Puis, avec l'appui des municipalités, le mouvement se répand dans d'autres villes telles que Villa Clara, Matanzas, Cienfuegos, Santiago de Cuba.

L'Etat soutient aussi les agriculteurs ou les jardiniers à travers l'assistance technique ou matérielle et l'envoi de conseillers qui les éduquent aux techniques de l'agro-écologie et à l'utilisation de méthodes biologiques, car la législation interdit l'utilisation en ville de produits chimiques pour la production agricole de petite échelle, exigeant des connaissances spécifiques, que la population ne maîtrise pas.

Nombre de techniques agricoles en général et de l'agro-écologie en particulier sont inconnues de la plupart des nouveaux jardiniers, citadins, de professions diverses, qui expérimentent pour la première fois l'agriculture. Dans le cas de potagers urbains installés par d'anciens travailleurs des fermes d'Etat, ces derniers possèdent des connaissances propres aux systèmes d'exploitation de monoculture à grande échelle fortement utilisatrice d'intrants chimiques. C'est la raison pour laquelle le soutien de l'Etat est indispensable au développement de l'agriculture urbaine.

Le département de l'agriculture urbaine facilite aussi l'octroi de terres inutilisées aux citadins qui veulent les mettre en activité pour produire de la nourriture. L'Etat légifère en faveur de la remise de terres en production alimentaire et autorise l'installation de points de vente de produits agricoles dans ces mêmes jardins.

L'agriculture urbaine prend à Cuba une telle importance que l'Etat crée en 1997 le Groupe national pour l'agriculture urbaine (GNAU), coordonné par l'Institut de recherche fondamentale en agriculture tropicale (Inifat), et met en place un nouveau programme d'agriculture urbaine formé de vingt-huit sous-programmes de production de légumes frais, de plantes médicinales, d'arbres fruitiers, de matière organique, etc. (Gonzalez Novo, Castellanos, Price, 2010 ; Febles-González et al., 2011). Parmi les différents sous-programmes, celui qui concerne la production de légumes et de condiments frais connaît une importante évolution, multipliant par mille la production entre 1994 et 2005 (Companioni Concepción, Rodriguez Nodals, 2006).

\section{Une diversité de formes d'organisations}

Il existe de nombreuses formes d'agriculture urbaine selon les méthodes agricoles de production développées et le type d'organisation sociale 
ou productive adopté, tels les huertos populares, autoconsumos, patios, parcelas familiares ou encore les organopónicos ou huertos intensivos (Altieri et al., 1999) ${ }^{(6)}$.

Les huertos populares, les patios et les parcelas familiares sont les structures les plus répandues dans l'agriculture urbaine avec le plus grand nombre de participants. Ce sont des jardins individuels ou partagés par des groupes de voisins sur des terres vacantes octroyées par l'Etat ou des agriculteurs particuliers qui cultivent leurs petites parcelles de terres privées (terrasses, cours, etc.). Pour la plupart, ces jardiniers s'organisent de manière collective

(6) Nous ne citerons pas dans cet article les fincas suburbanas, trop récemment incorporées dans le programme d'agriculture urbaine à Cuba pour que nous ayons le recul nécessaire à leur analyse. à travers la création de grupos de parceleros et horticultores. Ces groupes facilitent l'échange d'information et de connaissances entre jardiniers et servent d'intermédiaires au Minag pour la distribution de ressources agricoles et la réalisation d'ateliers avec les techniciens gouvernementaux. Ainsi, les patios et parcelas représentent une forme d'agriculture communautaire et familiale dont la production est destinée principalement à l'autoconsommation et dont le travail repose sur la main-d'œuvre de la famille. Les autoconsumos sont des jardins appartenant aux institutions publiques telles que les écoles, les cafétérias, les usines ou les hôpitaux, et dont le but est de produire des aliments pour approvisionner les cantines de ces institutions. Les éventuels surplus de production sont vendus aux travailleurs de ces institutions ou au public en général (Douzant-Rosenfeld, 1999).

Enfin, il existe au sein de l'agriculture urbaine à Cuba des méthodes spécifiques de jardinage intensif, telles que les huertos intensivos, des jardins sur des terres cultivables où s'incorpore de la matière organique, ou les organopónicos, des potagers horticoles organisés en "plates-bandes plantées sur un mélange de terre et de matière organique» (Argaillot, 2014). Ces derniers ont été créés avec l'appui de l'Etat à la fin des années 90, au sein des autoconsumos, des coopératives, ou par les agriculteurs privés. Ils sont idéals pour cultiver sur les sols très pauvres ou dégradés des espaces urbains.

Même si l'installation des organopónicos est financée par l'Etat, les travailleurs en ont l'entière gestion (Douzant-Rosenfeld, 1999). Les organopónicos sont une des formes les plus productives d'agriculture urbaine: ils peuvent atteindre des rendements de plus de 20 kilos par mètre carré. Ils permettent de cultiver tout au long de l'année des cultures maraîchères en rotation qui sont vendues librement à la population, mais à des prix de vente fixés par l'Etat. Ils offrent au public une grande variété de produits, généralement non inclus dans le carnet de rationnement, tels que des laitues, des épinards, du basilic, des oignons de printemps, des tomates, des petits pois, etc., à des prix plus bas que ceux du marché libre paysan.

Les agriculteurs urbains s'organisent, s'ils le souhaitent, en coopératives de crédit et services (CCS) ou en unidades básicas de producción cooperativa (UBPC). Les premières représentent une forme d'organisation dans laquelle les agriculteurs possèdent leurs propres parcelles de terre de manière individuelle et se regroupent pour l'achat de matériel et la commercialisation de leurs produits (Marzin, 2013). Les secondes sont une forme d'organisation constituée par des ouvriers agricoles qui reçoivent des terres de l'Etat en usufruit pour un temps indéfini. Celles-ci furent créées lors de la période 
spéciale à Cuba afin de remplacer les fermes d'Etat par un système plus efficace et moins centralisé (Escaith, 1999). Au sein des CCS ou des UBPC, le processus de prise de décision est démocratique, l'adhésion est volontaire et l'entière gestion est entre les mains des producteurs. Cependant, l'autonomie se voit parfois limitée, notamment dans le cas des UBPC, car elles doivent en certaines occasions se soumettre aux directives de l'Etat en matière productive, technologique ou d'investissement. Elles conservent toutefois leur autonomie de gestion, et les membres des UBPC sont les propriétaires de la totalité de la production (Rodríguez Membrado, López Labrada, 2011). Il existe des expériences remarquables d'UBPC avec un degré élevé d'autonomie, notamment dans la fixation des objectifs de production. Le cas le plus connu est l'Organopónico Vivero Alamar, fondé en 1997 à La Havane sur 800 mètres carrés de terres. Cette UBPC, composée à l'origine de cinq membres, en intègre aujourd'hui cent quatre-vingts. Sur une surface actuelle légèrement supérieure à 10 hectares, elle a produit 300 tonnes de légumes en 2013 (pour 239 tonnes en 2006 et 20 tonnes en 1997), vendus directement à la population ou à des centres sociaux à des prix accessibles (FAO, 2014; Fernández Domínguez, Cruz Reyez, Arteaga Hernández, 2007). La direction démocratique et l'autonomie de gestion à l'égard de l'Etat cubain ont joué un rôle essentiel dans le succès de cette UBPC.

\section{Consolidation du mouvement}

Tout au long de la période spéciale, le mouvement de l'agriculture urbaine gagne de l'ampleur. A Cuba, en 1996, il existe 1613 organopónicos; à La Havane, en 1997, on recense plus de 5000 huertos populares, regroupant 26604 jardiniers sur 1854 hectares et, en 1998, 366 autoconsumos sur 6365 hectares de terre. Ainsi, plus de 100000 emplois sont créés dans les années 2000 par l'agriculture urbaine (Hernandez, 2006).

Cette agriculture devient la principale source de légumes frais pour la population urbaine. Elle représente $60 \%$ du total des légumes consommés à Cuba à la fin des années 90. En 2000, la moitié du riz consommé à Cuba en est issu, de même que 70 \% des légumes ou encore 39 \% des fruits (agrumes exceptés). Certains jardins élèvent aussi des animaux pour la production d'œufs, de lait et de viande. L'agriculture urbaine devient alors un pilier de la sécurité alimentaire de la nation (Rodriguez Botella, 2010), et un régime alimentaire plus varié s'installe sur la table des Cubains, plus riche en calories, en protéines, en vitamines et en minéraux. Ainsi, l'agriculture urbaine contribue à l'actuelle consommation moyenne de 3547 calories par jour.

La production urbaine se voit de plus stimulée par la décentralisation de la commercialisation avec l'autorisation des marchés libres paysans en 1994, où les agriculteurs peuvent vendre leur production directement aux consommateurs et améliorer ainsi l'économie familiale. En outre, les jardiniers peuvent vendre leur production directement depuis leurs jardins sans payer d'impôts ou depuis les points de vente situés à moins de 5 kilomètres des unités productives, réparties dans tous les quartiers, ce qui constitue un essor remarquable des circuits courts de consommation.

La vente sur les marchés est pourtant rarement la motivation principale, car les jardiniers donnent en général la priorité à la consommation familiale, 
celle des amis proches et des voisins aux faibles ressources. De plus, certains jardiniers établissent des contrats directs avec les écoles, les hôpitaux, les maisons de retraite et les centres sociaux de leurs communautés pour les approvisionner en produits agricoles issus de l'agriculture urbaine (Argaillot, 2014).

Selon la FAO (2014), les ventes de l'agriculture urbaine à La Havane ont été de 58000 tonnes en 2013 dont 26000 ont été conclues dans les points de vente et 6000 à travers des contrats avec les centres de santé, les écoles et autres institutions publiques à caractère social.

Enfin, une multiplicité d'acteurs, au-delà des agriculteurs eux-mêmes, contribuent au succès de l'agriculture urbaine à Cuba, tels que la red de fincas municipales de semillas (réseau des fermes municipales de semences), les centros reproductores de entomófagos y entomopatógenos (centres de production des entomophages et entomopathogènes) et les centros de materia orgánica (centres de matières organiques), qui réduisent la dépendance aux ressources externes.

Ainsi, au vu des résultats positifs de l'agriculture urbaine, le mouvement gagne des adeptes au cours des années 90 et cesse d'être une réponse à la crise pour devenir une pratique durable sur le long terme. En 2013, on comptabilise plus de 4000 organopónicos (organoponiques), plus de 6000 huertos intensivos (jardins intensifs) et une production de légumes et de plantes aromatiques de 1158952 tonnes (contre 964869 en 2012). De cette façon, de nombreuses villes deviennent plus autonomes en réduisant leur dépendance envers les zones rurales pour leur alimentation (tableau 1).

L'agriculture urbaine met à profit l'esprit collectif des Cubains et l'importance de la coopération comme valeur sociétale à Cuba. Le rôle de soutien joué par l'Etat est ici remarquable et permet d'élargir et de consolider ce mouvement d'origine populaire à une plus grande échelle.

De ce fait, parmi les multiples bénéfices de l'agriculture urbaine à Cuba nous pouvons citer : la création de nouveaux emplois, l'augmentation des revenus des ménages, l’embellissement des villes, l'accroissement de la biodiversité,

\section{Tableau 1}

\section{Base productive de l'agriculture urbaine à Cuba*}

\begin{tabular}{|c|c|c|c|c|}
\hline & \multicolumn{3}{|c|}{ Cuba } & \multirow{3}{*}{$\begin{array}{c}\text { La Havane } \\
2013 \\
\text { Unités }\end{array}$} \\
\hline & \multirow{2}{*}{$\begin{array}{c}1995 \\
\text { Unités }\end{array}$} & \multicolumn{2}{|c|}{2013} & \\
\hline & & Unités & Hectares & \\
\hline Organopónicos & 1631 & 4014 & 1956,2 & 97 \\
\hline Huertos intensivos & 429 & 6405 & & 318 \\
\hline Patios & & 385311 & 12983 & 89000 \\
\hline Parcelas & 26604 & 145626 & 31124 & 5100 \\
\hline
\end{tabular}

* Les autoconsumos font partie de l'agriculture urbaine, mais nous ne les incluons pas dans la présente analyse, car notre objectif est l'étude des formes productives autonomes par rapport au pouvoir étatique.

Source: élaboration de l'auteur d'après Companioni Concepción (2013), FAO (2014) et Argaillot (2014). 
l'incorporation de pratiques agricoles plus respectueuses de l'environnement, l'amélioration de la sécurité alimentaire, la création de liens plus étroits entre agriculteurs et consommateurs, etc. Elle contribue également à la souveraineté alimentaire d'un pays historiquement dépendant des importations pour assurer sa propre alimentation.

\section{L'agriculture urbaine: nouvel acteur de l'économie solidaire cubaine}

L'émergence de l'agriculture urbaine à Cuba représente non seulement une nouvelle source d'approvisionnement en aliments pour la population, mais aussi une organisation économique alternative du secteur agricole face au traditionnel système économique centralisé et verticaliste du socialisme cubain.

La disparition du socialisme d'Europe de l'Est et le recentrage de l'Etat sur les besoins fondamentaux de la société favorisent en effet l'émergence de nouvelles forces productives ayant comme moteur les communautés, qui jouent un rôle prédominant pour pallier les effets de la crise.

L'agriculture urbaine dessine ainsi une forme de production alternative, qui ne se limite pas au dilemme entre insertion de l'île à l'économie capitaliste globale et continuité d'une institutionnalisation étatique centralisée et régulatrice de l'économie. Elle permet au contraire d'envisager la consolidation d'un secteur non étatique d'unités économiques autogérées offrant un fort potentiel pour la rénovation du système économique cubain (Betancourt, Sagebien, 2013).

Cette nouvelle forme d'économie sociale se différencie des entreprises capitalistes dont l'objectif est la concentration de pouvoirs et l'accumulation de richesses et de capital à travers l'exploitation du travail et de la nature, mais aussi des traditionnelles entreprises étatiques cubaines au sein desquelles les initiatives individuelles et la créativité des travailleurs sont limitées. L'agriculture urbaine est en revanche guidée par une rationalité différente de l'entreprise capitaliste: une rationalité solidaire orientée vers la création de valeurs d'usage, et non d'échange, et le bien-être de la communauté, car la production est destinée à la satisfaction des besoins de la famille et de la communauté, et non à l'obtention de profit et à l'accumulation de capital sans limite (Coraggio, 2002). En d'autres termes, une économie créatrice de société.

Les acteurs de l'agriculture urbaine à Cuba se caractérisent par la prédominance d'une logique de réciprocité, de solidarité, de coopération et d'horizontalité des rapports humains (Chaguaceda Noriega, 2011). Des actions telles que le don, l'aide entre voisins ou la répartition des aliments mettent en évidence cette logique solidaire au sein des expériences d'agriculture urbaine. De même, elle promeut des pratiques plus respectueuses de l'environnement et une utilisation plus rationnelle des ressources de chaque territoire.

L'agriculture urbaine permet aussi au pays de revaloriser le travail et la vie liée à la terre, de stimuler l'autogestion comme forme de production, de promouvoir la gestion directe dans la résolution des problèmes 
et la satisfaction de besoins sociaux, de renforcer les liens entre les personnes au sein de la communauté par sa logique solidaire et de consolider les communautés comme structures d'autorité collective (Garcia Guerreiro, 2012). Cependant, pour permettre l'expansion et la consolidation de ces unités économiques non étatiques responsables et solidaires, la transformation du rôle de l'Etat est fondamentale afin de laisser plus de place à l'autonomie de gestion.

\section{L'autogestion dans la construction du socialisme}

Parmi les principaux défis dans la rénovation du socialisme cubain figurent en première ligne une plus grande autonomie de gestion et une décentralisation des unités économiques. Car l'ordre institutionnel montre encore les signes d'un haut degré de centralisation et de tendances bureaucratiques qui limitent l'initiative populaire au sein des entreprises étatiques.

C'est la raison pour laquelle, malgré l'essor de formes de production non étatiques et l'appui matériel et symbolique de l'Etat aux structures telles que les coopératives, beaucoup d'entre elles se retrouvent subordonnées à la planification étatique. C'est le cas notamment des UBPC, créées pour démanteler les fermes d'Etat et dont l'efficacité est parfois limitée, parce qu'elles doivent se subordonner aux décisions de l'Etat (Nova, 2009).

Cependant, les impacts positifs et les résultats remarquables des initiatives d'agriculture urbaine sous un mode de direction collectif et démocratique dans la création d'emplois et la génération de valeurs d'usage mettent en évidence la nécessité de leur laisser plus de place dans l'économie cubaine.

Le régime cubain pourrait donc s'appuyer sur les valeurs et les principes de l'économie sociale et solidaire et sur les expériences de l'agriculture urbaine à Cuba pour dépasser la tradition étatiste et la bureaucratisation du système, sans pour autant revenir vers l'économie de marché, et avancer ainsi vers l'approfondissement du socialisme cubain sur les bases de l'autogestion et donc de la participation des travailleurs dans la gestion des entités productives auxquelles ils appartiennent.

L'élargissement de pratiques de travail associatif autogéré permettrait le développement des capacités individuelles et collectives, la promotion de formes de travail non salarial et la complémentarité de la redistribution par une autorité centrale avec l'appropriation et la redistribution au sein des unités économiques. Il semble alors indispensable d'élargir l'expérience autogérée de l'agriculture urbaine à d'autres secteurs productifs comme l'industrie ou les services.

Enfin, le développement de l'autogestion à Cuba est fortement favorisé par la culture populaire propre de l'île, qui revendique les valeurs de solidarité, d'équité et de dignité. Cela facilite la redéfinition de l'organisation économique du pays vers plus d'autonomie des unités économiques.

\section{Redéfinition du rôle de l'Etat}

La consolidation des nouvelles formes d'organisations économiques, sociales et solidaires implique une redéfinition des principes d'organisation du travail et de coordination-planification, références absolues de l'économie publique, afin de donner plus de place à l'expérimentation responsable de formes 
de travail autonome, de planification communautaire (Coraggio, 2011) et d'auto-organisation du voisinage.

L'entreprise étatique et la direction centralisée ont joué en effet un rôle prédominant dans le système économique cubain de la révolution, avec une faible participation des communautés dans la gestion de projets économiques locaux. A l'opposé, l'agriculture urbaine a permis l'ouverture des espaces de participation populaire et la décentralisation des fonctions de l'Etat, ainsi que la maximisation de l'utilisation de ressources locales. Néanmoins, la modification de la matrice productive du pays ne signifie pas que l'Etat doive s'effacer. Au contraire, il doit conserver sa fonction dans la redistribution progressive des ressources et la canalisation des investissements prioritaires pour le développement du pays. L'Etat doit aussi continuer à garantir l'accès universel à la santé et à l'éducation et veiller au maintien d'un faible niveau d'inégalités.

L'importance de l'Etat dans la redistribution des ressources est mise en évidence dans le cas de l'agriculture urbaine, dont le succès est dû à la créativité du peuple cubain, ainsi qu'à l'implication de l'Etat, qui a offert des terres et l'assistance technique et matérielle. La décentralisation de la commercialisation joue également un rôle décisif.

Cependant, pour garantir la réussite de cette décentralisation des fonctions de l'Etat et la consolidation d'un système économique sur la base de l'autogestion promouvant le respect des droits fondamentaux, il est indispensable que les unités économiques non étatiques soient responsables socialement, privilégient la création de valeurs d'usage et se préoccupent du bien-être collectif et du développement local.

Sans solidarité envers la communauté des entreprises non étatiques, l'autogestion comme système de gestion économique semble insuffisante pour garantir la réussite des transformations économiques à Cuba, car ces unités économiques pourraient se concentrer sur leurs intérêts particuliers (l'intérêt individuel du collectif autogéré) plutôt que sur les intérêts et les besoins de la société. Ainsi, l'action de l'Etat serait complémentaire des actions des acteurs économiques autonomes, responsables et autogérés qui partagent avec lui le projet socialiste. Le système socialiste cubain pourrait ainsi, selon les termes de Chaguaceda Noriega (2011), "révolutionner la révolution de l'intérieur » grâce au développement d'organisations sociales autogérées basées sur les principes de la réciprocité, de la coopération, de la solidarité et de la responsabilité collective (Coraggio, 2011).

\section{Autosuffisance, territorialité et développement local}

L'émergence de l'agriculture urbaine à Cuba met en lumière l'importance de la décentralisation territoriale de l'Etat et de la gestion locale dans l'organisation économique. Il semble en effet indispensable de donner plus d'autonomie politique aux communautés locales afin de les impliquer et de leur permettre de prendre en main leur destin. Le contrôle accrû du processus de production par la communauté, comme dans le cas de la production et de la commercialisation de produits agricoles en ville, permettrait aux citoyens de gérer directement les nécessités économiques et sociales locales et d'œuvrer ainsi au bien-être de leur communauté. 
En outre, l'expérience de l'agriculture urbaine à Cuba contribue notablement à l'augmentation des niveaux d'autarcie locale, entendue comme la capacité de la communauté à satisfaire ses besoins élémentaires avec des ressources propres (Coraggio, 2011): la production de l'agriculture urbaine est destinée à la satisfaction des besoins alimentaires des habitants du quartier, et des intrants tels que des semences, du compost, des biopesticides, etc. sont produits localement, ce qui favorise l'autonomie des communautés (Companioni Concepción, 2013). Les capacités propres du territoire sont ainsi mobilisées pour susciter un développement territorial endogène capable de répondre aux besoins fondamentaux de la société en surpassant le paternalisme traditionnel (Betancourt, Sagebien, 2013). C'est pourquoi la redéfinition de l'organisation économique à Cuba exige du gouvernement le soutien aux unités économiques associatives, facteur de développement local.

Pour autant, compte tenu de l'impossibilité d'une autarcie complète, il est nécessaire de maintenir la réciprocité intercommunautaire et la redistribution des ressources par l'autorité centrale légitime, comme c'est le cas de l'agriculture urbaine.

Face à la planification centrale de l'Etat, l'agriculture urbaine permet une expansion des espaces d'expérimentation populaire responsable, ainsi que le renforcement du tissu social à travers la création de relations de proximité, la valorisation de l'espace local et l'accent mis sur la responsabilité sociale. Elle permet, enfin, la participation de la communauté à la construction de nouvelles formes de production, de distribution, d'échange et de consommation des aliments et la réorganisation du pouvoir centralisé vers des espaces de pouvoir collectif communautaire.

\section{Conclusion}

L'effondrement de l'Union soviétique a entraîné un changement radical du système agricole cubain vers un système d'agriculture local, diversifié, de proximité, plus respectueux de l'environnement et promoteur de relations de confiance entre producteurs et consommateurs.

Grâce à l'agriculture urbaine, le pays est parvenu à augmenter la production domestique de produits alimentaires et donc la sécurité alimentaire, à réduire les coûts de transport et la consommation de pétrole, à accroître la variété des produits pour la consommation des ménages, à garantir l'accès des citadins aux produis frais et de bonne qualité, à élargir les pratiques d'agriculture biologique et d'agro-écologie, à développer la biodiversité, à transformer des terrains vagues en lieux de production agricole, à augmenter les revenus des ménages, à créer de nouveaux emplois, etc.

Par conséquent, le cas cubain peut constituer une source d'inspiration pour d'autres pays en termes de lutte contre la faim, de création d'un système agricole plus résilient, de protection de l'environnement et de construction d'un modèle agricole moins dépendant de ressources non renouvelables; en d'autres termes, de construction de systèmes sociotechniques de production alimentaire différents du modèle dominant.

La réussite de l'agriculture urbaine à Cuba est également due à l'appui de l'Etat, qui a opté pour une politique active de distribution de terres pendant 
la période spéciale et de soutien aux mouvements spontanés de la population visant à produire leurs propres aliments. L'Etat accompagne aussi les agriculteurs urbains à travers les programmes de formation et facilite l'accès aux semences, à la terre et aux crédits.

Cependant, il ne s'agit pas seulement de distribuer des ressources, mais aussi de garantir des espaces plus importants d'autonomie pour les initiatives familiales, associatives et communautaires, en redéfinissant le rôle de l'Etat comme coordinateur d'actions dans le territoire.

De même, l'ouverture de nouveaux espaces de participation populaire et d'expérimentation responsable dans d'autres secteurs économiques tels que les services, la construction ou l'industrie semble indispensable, mais, pour cela, il est fondamental que l'Etat cubain reconnaisse les acteurs de l'économie sociale et solidaire comme de véritables acteurs socioéconomiques pour l'ensemble des activités économiques.

Selon Coraggio (1999), cette reconnaissance équivaut à la combinaison de l'économie publique avec une économie plus centrée sur le travail associatif autogéré, et donc à l'action conjointe de l'Etat et de la société organisée dans la production de biens publics.

Enfin, ces initiatives présentent un fort potentiel pour la rénovation du système productif cubain sur les bases de l'autonomie de gestion, de la socialisation des résultats et de la direction collective et démocratique, mais aussi pour l'approfondissement de la démocratie grâce à la participation de la société civile dans l'élaboration, l'approbation et l'application de politiques publiques. Elles permettent ainsi d'envisager la construction d'une nouvelle matrice de production dans l'île avec des acteurs économiques qui n'appartiennent ni à l'économie publique ni à l'économie de marché, mais à l'économie sociale et solidaire, dont la finalité est le bien-être de la société sous des formes d'organisation autogérées, associatives et démocratiques engagées pour le bien-être de leurs communautés. 


\section{BiBLIOgRAPHIE}

Altieri M., Companioni N., Cañizares K., Murphy C., Rosset P., Bourque M., Nicholl C., 1999, "The greening of the "barrios": Urban agriculture for food security in Cuba ", Agriculture and human values, vol. $16, \mathrm{n}^{\circ} 2$, juin, p. 131-140.

Argaillot J., 2014, «Emergence et impacts de l'agriculture urbaine à Cuba », Espaces et Sociétés, nº 158, p. 101-116.

Betancourt R., Sagebien J., 2013, « Para un crecimiento inclusivo: empresas no estatales responsable en Cuba », Temas, n ${ }^{\circ}$ 75, p. 58-65.

Chaguaceda Noriega A., 2011, "Socialismo de Estado y asociacionismo en la Cuba actual ", in Bernal Gomez B. (dir.), Cuba hoy: perspectivas de cambio? México DF, Universidad autónoma de México, p. 321-340.

Companioni Concepción N., Rodriguez Nodals A., 2006, "Situacion actual, perspectivas y restos de la agricultura urbana en Cuba ", Agricultura Urbana, $\mathrm{n}^{\circ}$ 2, Actaf, p. 4-5. Companioni Concepción N., 2013, « Panorama historico y desarrollo actual de la agricultura urbana y suburbana en Cuba », Mémoires du séminaire international d'agriculture urbaine et périurbaine, réunions lors du IV congrès d'agriculture tropical, Convention Tropique, La Havane, Cuba, du 14 au 17 mai.

Coraggio J. L., 1999, Politica social y economía del trabajo, éd. Miño y Dávila, Madrid.

Coraggio J. L., 2002, « Hacia un proyecto de economía social centrada en el trabajo: contribuciones de la antropología económica", communication présentée lors du «Seminario Internacional PEKEA, un saber político y ético para las actividades económicas", 10-14 septembre 2002, Cepal, Santiago du Chili. Coraggio J. L., 2011, Economía social y solidaria: el trabajo antes que el capital, éd. Alberto Acosta y Esperanza Martínez, Quito, Abya Yala, p. 345-414.

Douzant-Rosenfeld D., 1999, "Les défis des réformes dans l'agriculture cubaine ", ¿Cubasi, Cuba no? Cahiers des Amériques latines, $\mathrm{n}^{\circ}$ 31-32, p. 161-181.
Escaith H., 1999, "Cuba pendant la période spéciale: ajustements ou transitions? », ¿Cuba si, Cuba no? Cahiers des Amériques latines, $\mathrm{n}^{\circ}$ 31-32, p. 35-53. Disponible sur Iheal. univ-paris3.fr/fr/publications.

FAO, 2014, «Informe de la FAO sobre la agricultura urbana y periurbana en la region ", Ciudades mas verdes en América latina, Rome, p. 10-19.

Febles-González J. M., Tolón-Becerra A., Lastra-Bravo X., Acosta-Valdés X., 2011, "Cuban agricultural policy in the last 25 years: From conventional to organic agriculture ", Land use policy, vol. 28, p. 723-735.

Fernández Domínguez P., Cruz Reyez J., Arteaga Hernández C., 2007, Cuatro experiencias exitosas en UBPC, Actaf, La Havane, p. 54-64.

Garcia Guerreiro L., 2012, « Aportes para una economía de la vida, aprendizajes desde los mundos campesinos », in Marañón-Pimentel B. (dir.), Solidaridad económica y potencialidades de transformación en América latina, Clacso, Buenos Aires, p. 181-207.

Gonzalez C., 2003, "Seasons of resistance: Sustainable agriculture and food security in Cuba », Tulane environmental law journal, vol. 16, p. 685-732.

Gonzalez Novo M., " Institucionalización de la agricultura urbana en la ciudad de La Habana ", rapport présenté lors du séminaire régional "Agricultura urbana en las ciudades del Siglo XXI », Quito, Equateur, avril 2000.

Gonzalez Novo M., Castellanos A., Price J., 2010, Testimonios agricultura urbana en ciudad de La Habana, Ipes-Actaf-Oxfam, La Havane, Cuba.

Guevara M. de los A., 2009, "Cuba: reforma y transformación agraria. La crisis de los noventa y el proceso de desestatalización de la agricultura ", Revista IDeAS, vol. 3, n 1 , janvier-juin, p. 6-29.

Gutelman M., 1963, «L'agriculture cubaine: la réforme agraire et les problèmes nouveaux ", Etudes rurales, $\mathrm{n}^{\circ}$ 8, janvier-mars, p. 62-83. 
Gutelman M., 1965, « L'agriculture cubaine en 1964 », Etudes rurales, n 19, p. 5-31.

Gutelman M., 1967, L'agriculture socialisée à Cuba: enseignements et perspectives, éd. François Maspero, Paris.

Habel J., 2003, "Cuba: les défis du nouveau "modèle" ", Revue Tiers Monde, n 173, p. 127-148.

Hernandez L., 2006, " La agricultura urbana y caracterización de sus sistemas productivos y sociales, como via para la seguridad alimentaria en nuestras ciudades ", Cultivos tropicales, vol. $27, \mathrm{n}^{\circ} 2$, Instituto nacional de ciencias agricolas, Cuba, p. 13-25.

Herrera R., 2005, Réflexions sur l'économie cubaine, Maison des sciences économiques, Paris.

Karnoouh L., 2007, «A propos de la permanence et du changement dans la Cuba contemporaine: un essai sur la période spéciale, Outre-Terre, vol. 1, n 18 , p. 337-344.

Marzin J., 2013, «Les leçons et les enjeux du mouvement coopératif agraire à Cuba », Recma, $\mathrm{n}^{\circ} 328$, p. 63-74.

Medea B., Collins J. et Scott M., 1987, Cuba:les enjeux de l'agriculture et de l'alimentation, L'Harmattan, Paris.

Murphy C., 1999, « Cultivating Havana: Urban agriculture and food security in the years of the crisis ", Development Report, $\mathrm{n}^{\circ} 12$, Institute for food and development policy-Food First, Oakland.

Nova A., 2001, « La agricultura cubana previo a 1959 hasta 1990 ", Transformando el campo cubano: avances de la agricultura sostenible, La Havane, Actaf, p. 1-14.

Nova A., 2009, "Línea de desarrollo y resultados de la agricultura cubana en los últimos 50 años », Evento XX Aniversario du CEEC, seminario sobre economía cubana y gerencia empresarial, Centro de estudios de la economía cubana, La Havane, 27-29 mai 2009.

Raymond P., 2002, « Vers une nouvelle orientation pour l'agriculture cubaine? », in Herrera Rémy (dir.), Revue Tiers Monde, Trajectoires latino-américaines, Regards sur Cuba, vol. 43Oे, $\mathrm{n}^{\circ} 171$, p. 579-598.

Rodriguez Botella E., 2010, «Cuba’s alternative/inward-looking development policies. Changing production patterns and land decentralization: Toward sustainable small farming (1990-2008) », Sociedad española de historia agraria, documentos de trabajo, DT-Seha $\mathrm{n}^{\circ} 10-11$.

Rodríguez Membrado E., López Labrada A., 2011, « La UBPC: forma de rediseñar la propiedad estatal con gestión cooperativa », in Piñeiro Harnecker Camila (dir.), Cooperativismoy socialismo: una mirada desde Cuba, La Havane, éd. Caminos, p. 337-365.

Rosset P., Medea B. (éd.), 1994, The Greening of the revolution:Cuba's experiment with organic agriculture, Ocean Press, Melbourne.

Roux M., 1999, «L'intendance quotidienne à La Havane dans les années 1990 ", ¿ Cuba si, Cuba no? Cahiers des Amériques latines, $\mathrm{n}^{\circ} 31-32$, p. 124-145. 